This is the final peer-reviewed accepted manuscript of:
A. Cicchella, M. Zini, M. Paolini, P. Tiberini, C. Stefanelli

Cycling training effects on fat metabolism blood parameters

Gazzetta Medica Italiana - Archivio per le Scienze Mediche, 2020 v.179(3)

The final published version is available online at:

http://dx.doi.org/10.23736/S0393-3660.19.04022-1

Rights / License:

The terms and conditions for the reuse of this version of the manuscript are specified in the publishing policy. For all terms of use and more information see the publisher's website.

This item was downloaded from IRIS Università di Bologna (https://cris.unibo.it/)

When citing, please refer to the published version. 


\section{Cycling training effects on fat metabolism blood parameters.}

2 Antonio Cicchella ${ }^{1}$, Maddalena $\mathrm{Zini}^{2}$, Moreno Paolini ${ }^{2}$, Paolo Tiberini ${ }^{1}$ and Claudio Stefanelli ${ }^{1}$

$3 \quad{ }^{1}$ Department for Quality of Life Studies, Rimini (Italy)

$4 \quad$ 2Dipartimento di Biochimica "Moruzzi”, Bologna (Italy)

5 Corresponding author: Antonio Cicchella, Department for Quality of Life Studies, University of

6 Bologna, Rimini, Corso d' Augusto 237, 47921 Rimini, Italia. E-mail: antonio.cicchella@unibo.it.

\section{Abstract}

8 Background: study the acute and middle term (4 weeks training) effect of cycling training on fat 9 blood hematological parameters, urine, fatigue, and general health in recreational well-trained cyclists.

Methods: 19 cyclists undergone 5 blood sampling: 1) before and after an incremental maximal ramp test 7 days before day $0(\mathrm{D}-0) ; 2$ ) before and after 1 hour exhaustion trial test at baseline (D-0) and after 28 days of training (D-28). Age 34,5 years $( \pm 9,5)$; weight $74,87 \mathrm{~kg}( \pm 6,6)$; height $177,3 \mathrm{~cm}$ $( \pm 5,2)$; BMI 26,3 $( \pm 4,9)$; VO2max 53,75 ml/kg/min $( \pm 6,01)$; km week $314,7 \mathrm{Km}( \pm 137,1)$.

Results: Acute effect was strong elevating WBC from $6,27 \pm 2,3410^{3} / \mathrm{ul}$ to $9,01 \pm 3,6310^{3} / \mathrm{ul}$, an increase in LDL and Total CHOL, in this respect, existing literature is controversial. No changes in body weight or blood pressure was observed after 1 month of regular training albeit lipid profile significantly improved, as well as GOT.

Conclusions: effect of a short incremental bout of exercise was to temporary elevated all the blood parameters except $\mathrm{MCH}$ and MCHC. A month of intensive training (km week: 314,7 Km $\pm 137,1)$ significantly improved blood lipids profile with no permanent effect on $\mathrm{WBC}$, blood pressure or body weight, but improved post effort lactate concentration and fatigue perception. Hematuria is confirmed to be a rare occurrence in recreational cyclists. Data can be useful for training monitoring and comparisons with similar groups of athletes, where there is a lack of information in literature and for comparing exercise effects.

Key words: exercise tolerance, hematological test, lactate, urinalysis, cycling. 
Recreational cycling is a wide practiced recreational sport and with aging, the risk of cardiovascular diseases increase. One of the main aims of aerobic exercise is to lowering cardiovascular risk through improving blood lipids profile ${ }^{1}$. However, is not completely clear the effect of continuous exercise in lowering blood lipoproteins and even the effect on other blood and urine parameters in recreational sportsmen and surprisingly, there are very few studies on the topic with sometime conflicting results in the athletic populations ${ }^{2}$. In addition, few studies examined the alterations in many common or critical laboratory parameters in controlled (lab) conditions, using standardized and controlled protocols. Despite its diffusion, it exist few studies on the acute and long time effect of cycling in improving fat profiles of recreational cyclists, a very large category of practioners. At our knowledge, it exist only one study on the acute effect of cycling on blood lipids, that show after 90 min of cycling at $50 \%$ of $\mathrm{VO}^{2}$ peak, an increase in LDL and HDL cholesterol and a decrease in triglycerides (TRI), with no changes in total cholesterol ${ }^{3}$. The efficacy of different low volume (3040 minutes , 3 times per week at 50\% of HRR-heart rate reserve-for 12 weeks) training regimes in middle age male recreational bikers $(+60 \text { years old })^{4}$ has been studied. Results show positive effects on body weight reduction and systolic and diastolic blood pressure $(83 \pm 7 \mathrm{mmHg}$ vs $80 \pm 5 \mathrm{mmHg}$ and $140 \pm 8 \mathrm{mmHg}$ vs $135 \pm 11 \mathrm{mmHg}), \mathrm{CHOL},(216,3 \pm 4 \mathrm{mg} / \mathrm{dl}$ at baseline vs $210,9 \pm 4,3 \mathrm{mg} / \mathrm{dl})$ and LDL (120,6 $\pm 4,2 \mathrm{mg} / \mathrm{dl}$ vs $116,9 \pm 4,7 \mathrm{mg} / \mathrm{dl})$. Effect of aerobic exercise on lowering blood pressure has also been observed in non-athletic population undergone training ${ }^{5}$ while metanalysis showed a significant effect of aerobic training in comparison to no effect of resistance training in decreasing plasma lipids of $0,10-0,8 \mathrm{mg} / \mathrm{dl}$ in general population. Middle term decrease of CHOL, LDL, and TRI, with an increase in HDL of $0.05 \mathrm{mg} / \mathrm{dl}$ was also observed ${ }^{6}$. Other studies in young soccer players $^{7}$ showed a middle term a decrease in CHOL from $155,6 \pm 25,7 \mathrm{mg} / \mathrm{dl}$ to $151,5 \pm 8,7 \mathrm{mg} / \mathrm{dl}$, in TRI from $88,4 \pm 41,7 \mathrm{mg} / \mathrm{dl}$ to $87,9 \pm 43,79 \mathrm{mg} / \mathrm{dl}$, in LDL from $89,9 \pm 20,9 \mathrm{mg} / \mathrm{dl}$ to $86,2 \pm 10,11$ $\mathrm{mg} / \mathrm{dl}$ and in HDL-C from $51,4 \pm 8,4 \mathrm{mg} / \mathrm{dl}$ to $50,78 \pm 5,11 \mathrm{mg} / \mathrm{dl}$. A meta-analytic study ${ }^{8}$, evidenced high-intensity aerobic training resulted only in the improvement of high-density lipoprotein cholesterol. However, an explanation of these dissimilar results can be the non-homogeneity in the exercise load, kind of exercise, and level of participants. One acute effect of the exercise bout on the immune response was strong elevating white blood cells (WBC) from $6,27 \pm 2,34^{\star} 10^{3} / \mathrm{ul}$ to $9,01 \pm 3,63^{\star} 10^{3} /$ ul. When comparing prerace specimens with those within 4 hours after the marathon in 32 runners, WBC counts increased $\left(5.5 \pm 0.2{ }^{*} 10^{3} / \mathrm{ul} \text { to } 17.4 \pm 1,5^{\star} 10^{3} / \mathrm{ul}\right)^{9}$ (Siegel, et al., 2001) ${ }^{10}$. Hemoglobin depletion in urine is also a rare finding after effort ${ }^{11}$. Transaminases (GOT and GPT) has been indicated as markers of liver (GPT) and muscle (GOT) damage ${ }^{12}$, thus is important to assess their behavior with exercise. Few information exist in literature about the changes in other 
common blood and urine markers immediately after effort ${ }^{3}$. Our aims were to assess the acute (after a maximal short trial) and middle term (4 weeks, after 1 hour exhaustive endurance trial), modifications of hematological blood and urine parameters in recreational well trained recreational cyclist. We also want to assess the middle term effect of endurance training on basic health parameters of recreational cyclists, as rest heart rate, blood pressure and fatigue perception.

\section{Materials and methods}

19 amateur male well trained cyclists undergone 5 blood sampling: 1) before and after an incremental maximal ramp test till exhaustion 7 days before day 0 (D-0); 2) before and after 1 hour exhaustion trial test at baseline (D-0) and after 28 days of training (D-28). Mean age of the subjects was 34,5 years $( \pm 9,5)$, body weight $74,87 \mathrm{~kg}( \pm 6,6)$, height $177,3 \mathrm{~cm}( \pm 5,2)$, BMI $26,3( \pm 4,9)$, $\mathrm{VO}^{2} \max 53,75 \mathrm{ml} / \mathrm{kg} / \mathrm{min}( \pm 6,01), \mathrm{km}$ per week $314,7 \mathrm{~km}( \pm 137,1)$. The subjects can be classified as "recreational road cyclists" ${ }^{13}$ and ethical committee clearance was granted by Ethical committee of University of Bologna. The subjects filled a questionnaire aimed at assessing alcohol and caffeine usage, protein, carbo and fats and water consumption. Reported diet did not change over the observation period. Clinical anamnesis was performed in order to exclude any past or ongoing pathology, which could interfere with the trials and the absence of drugs intake. $\mathrm{VO}_{2} \max$ $(\mathrm{ml} / \mathrm{kg} / \mathrm{min})$ was assessed one week prior the D-0, with an incremental ramp test on the cycloergometer (Lode Corival, The Nederlands) with ramp of 20 watts per minute until exhaustion. The $\mathrm{VO}_{2}$ max was measured during $3 \mathrm{~min}$ at $\max$ intensity in the exhaustion trial. After the incremental test and the exhaustion trial, blood lactate $(\mathrm{mM} / \mathrm{L})$ was measured at 3, 6 and 9 minutes, and the highest value (usually observed at 6 minute) was retained.

Fatigue protocol at the cycloergometer consisted of a light warm up followed by: 10 min at $70 \%$ of $\mathrm{VO}^{2} \max ; 9$ minutes at $50 \% \mathrm{VO}^{2} \max +1 \min$ at $90 \% \mathrm{VO}^{2} \max \mathrm{x} 4$, ending with 3 min at $\mathrm{VO}^{2} \max$ for a total of 60 min exhaustion test. Every 10 minutes the subjects were asked to rate the effort using the Borg RPE scale ${ }^{14}$. Heart rate was measured during the 1-hour bout and in the 10 subsequent minutes. Lactate was measured at 3,6, and 9 min post effort, after the VO2max test (MAX) and after the fatigue protocols. In order to assess the intensity of the effort, the subjects were asked to rank the effort in the last 10 minutes of the exhaustion on a Borg 20 visual analog scale. The subject practiced the same protocol on road at least three times per week for the subsequent 28 days. Urine sampling were taken twice at rest before trials at D-0 and D-28. The following biochemical parameters were measured on fasting blood sample, collected, frozen and processed with standard laboratory methods. Parameters have been corrected for plasma volume changes. The parameters 
that was collected refer to White blood cell (WBC, 10^3/ul), a general marker of inflammation, Red Blood Cell concentration (RBC, 10^6/ul), Hemoglobin (HGB, g/dl) and Hematocrite (HCT, \%). Also, we considerate the Mean Corpuscular Volume (MCV, fl), Mean Content of Hemoglobin $(\mathrm{MCH}$, pg), Mean Concentration of Hemoglobin Content (MCHC, g/dl), Red Blood Cell Distribution wide (RDW, \%), Glicemy (mg/dl). Moreover, we measured a few parameters of organ function, as GPT (U/L), a marker used for liver and muscle damage; Transaminases YGT and GOT, marker of muscle damage ${ }^{12}$. Finally HDL-Cholesterol (mg/dl), total Cholesterol (CHOL, mg/dl), Triglycerides (TRI, mg/dl) and LDL-Cholesterol (LDL, mg/dl).

The following biochemical parameters were measured on urine: specific weight, $\mathrm{pH}$, Albumin, Sugar, Urobiline, Bilirubin, hemoglobin, Nitrates, Acetone, Leucocitary Esterase, deposits, leucocytes. In order to avoid hemoconcentration, subjects have been kept constantly hydratated checking they can drink water ab libitum during the 1-hour bout. Blood pressure was measured at rest 5 and 10 minutes after the cessation of the exhaustion trials at D-0 and D-28. Blood pressure at rest was in normal range for all the subjects according to their age. Statistical analysis was performed using a $\mathrm{T}$ test for paired samples with IBM-SPSS v.20 software, with significance level set at $0,5 \%$. All the subjects participating in the study gave their informed consent. Clearance was given by the University of Bologna ethical committee.

\section{Results}

Body weight remained unchanged over the 28 days in total group $(74,87 \pm 6,48 \mathrm{~kg}$ D-0, 74,64 $\pm 6,19$ $\mathrm{kg}$ D-28). $\mathrm{VO}^{2} \max$ measured during the last bout of 3 minutes at $\mathrm{VO}^{2}$ max during the fatigue protocol, improved significantly between D-0 and D-28 $(49,8 \pm 9,93 \mathrm{ml} / \mathrm{kg} / \mathrm{min}$ vs. $59,8 \pm 8,7$ $\mathrm{ml} / \mathrm{kg} / \mathrm{min} ; \mathrm{p}=0,22)$. In the incremental test performed one week before D-0, VO2max was 53,75 $\pm 6,01 \mathrm{ml} / \mathrm{kg} / \mathrm{min}$. Heart rate measured at minute 10 of the recovery, significantly decrease from D- 0 to $\mathrm{D}-28(105$ vs $99, \mathrm{p}=0.04)$. Blood pressure $(\mathrm{mm} / \mathrm{Hg})$ at 10 minutes post exercise didn't change significantly between D-0 and D-28 (max and min: $114 \pm 16 \mathrm{~mm} / \mathrm{Hg}$ and $82 \pm 13 \mathrm{~mm} / \mathrm{Hg}$ vs $120 \pm 14$ $\mathrm{mm} / \mathrm{Hg}$ and $82 \pm 11 \mathrm{~mm} / \mathrm{Hg}$ ).

Urine. Specific weight at D-0 was $1013,6 \pm 5,9 \mathrm{mg}$ and $1016 \pm 9,8 \mathrm{mg}$ at D-28, without any statistically significant differences and $\mathrm{pH}$ remained unchanged too $(5,85 \pm 0,76$ and $5,7 \pm 0,76)$ as well as albumin, sugar, bilirubin, hemoglobin, nitrates, acetone, leucocytary esterase, microscopic deposits, leucocytes, which remained absent, while urobiline remained stable at $0.2 \mathrm{mg} / \mathrm{dl}$ after 1 month training. Only one subject showed a presence in urine of HGB at 1 month confirm that hematuria is a relatively rare post exercise finding as previously observed ${ }^{11}$. The results of Borg's 
128 test indicate a close value to maximal intensity effort: D $019 \pm 1,5$ and D $2819 \pm 1$ score points, 129 without any significant difference. The higher value for lactate was retained and is reported in Table I. Maximum lactate concentration significantly decreased between D-0 and D-28.

[Table I]

Results for hematological parameter prior and after the incremental test are reported in Table II.

[Table II]

All parameters significantly increased except $\mathrm{MCH}$ and $\mathrm{MCHC}$.

Results for hematological parameters at D-0 and D-28 in the pre-trial conditions are shown in Table III.

[Table III]

In the graph 1, are shown the effects of the 28 days of training.

[Graph 1]

\section{Discussion}

All parameters show an increase pre/post trial except $\mathrm{MCH}$ and $\mathrm{MCHC}$. This result can indicate a lack of water shift into the erythrocytes during the trial, as demonstrated before ${ }^{15}$ : we can hypothesize the "ab libitum" water drinking during the trials, is not enough to guarantee water shift in the erythrocytes, which have a limited capacity to store water, not connected with water ingestion. We find an acute increase in LDL and CHOL, contrary to other studies in marathoners who found a decrease in LDL, and no change in cholesterol ${ }^{16}$, but according to another study which showed similar transient results in cyclists ${ }^{17}$. While on the long term the decrease of HDL, LDL and CHOL are in accord with others studies in young soccer players ${ }^{7}$. They show a decrease in CHOL from $155,6 \pm 25,7 \mathrm{mg} / \mathrm{dl}$ to $151,5 \pm 8,7 \mathrm{mg} / \mathrm{dl}$, in LDL from $89,9 \pm 20,9 \mathrm{mg} / \mathrm{dl}$ to $86,2 \pm 10,11 \mathrm{mg} / \mathrm{dl}$ and in HDL from $51,4 \pm 8,4 \mathrm{mg} / \mathrm{dl}$ to $50,78 \pm 5,11 \mathrm{mg} / \mathrm{dl}$. One acute effect of the exercise bout on the immune response was strong elevating WBC from $6,27 \pm 2,34^{\star} 10^{\wedge} 3 / \mathrm{ul}$ to $9,01 \pm 3,63^{\star} 10^{\wedge} 3 / \mathrm{ul}$, according to other studies ${ }^{9}$ which compared prerace specimens with those within 4 hours after the marathon in 32 runners who measured an increased WBC $(5.5 \pm 0.2$ to $17.4 \pm 1,5)$. We observed a significant increase of HGB and HCT only the acute measurements, while other studies in marathoners didn't observed hematocrit and hemoglobin changes ${ }^{10}$. Contrary to findings after a marathon run $^{18}$, in our study RBC and MCV increased, this seems a characteristic of cycling effort. Depending on the study, hematocrit decreased ${ }^{18},{ }^{19}$ not changed ${ }^{20}$ or increased ${ }^{10,21}$ after a marathon. Besides water loss, the increase in hematocrit can be attributed to the breaking of red blood cells in 
the foot's plantar circulatory bed during running ${ }^{22}$ and even if this is not the case of cycling, a certain degree of compression on the plantar surface of the feet is present. In addition, the controversial observations in previous studies showing an RBC (hemoconcentration) increase, hypothesized it can be due to differences in fluid intake and environmental conditions (e.g. hot). In our study, where subjects were allowed (and controlled) to drink ab libitum, we observed a significant acute increase in all hemoglobin parameters.

We observed on a long term a positive effect on the lipid profile and glycemic indexes, as confirmed in previous studies ${ }^{2}$. The acute increase of WBC observed pre-post trial at D-0, was not present after one month of training, being transitory. Probably a longer period is necessary to observe a lasting effect on WBC. It was observed ${ }^{23}$ an increase in WBC, which is an important indicator of immune function, after 1 year of training in recreational soccer players. Mean hemoglobin content also show an increase on the long term. GOT, MCV and MCH showed a significant difference at 1 month. Being GOT a marker of muscle damage ${ }^{12}$, a reduction in this parameter, indicate a better state of training.

\section{Conclusions}

Recreational cycling population is an at risk group for cardiovascular disease, mainly due to overweight. Accumulation of arterial cholesterol and triglycerides is a co-factor in heart diseases. For this reason and because is a low impact activity, cycling is highly recommended to middle aged men. Aging is also associated with increased visceral fat and body weight. There are few data in the literature about normal hematological values for this population of recreational sportsmen, and contradictory results on the changes of basic hematological parameters with training, despite the large diffusion of this recreational activity. In our study, we observed an acute post exercise increase of GLI, TRI, HDL-C and LDL-C and CHOL, WBC, and hematocrit, differently from other studies who show an increase in LDL, HDL and a decrease in TRI and no acute changes in CHOL in runners ${ }^{3}$. Endurance trials of running and cycling seems thus to have a different acute effect on acute blood fat turnover. On the long term, we observed a decrease in plasma CHOL, HDL and LDL, and a slight increment in the MCV and MHC, without any significant change in the others blood parameters. We did not observe, except in one case, hematuria, confirming it is a rare event as previously observed ${ }^{11}$. Metanalysis studies ${ }^{8}$ show that high-intensity aerobic training results in improvement only in high-density lipoprotein cholesterol in normal population. However, when considering athletic populations, the improvement affects all the lipid profile, and we confirm this finding. This result can be ascribed to the strong biochemical reactions triggered by training. We provided the evidence that recreational cycling has a beneficial long-term effect on the lipid profile in a different way respect to running, even if we did not observed any positive effect on further 
blood pressure reduction or fatigue perception, probably because our subjects have reached their optimal values. Cycling seems to differ from running in the effect on hematological parameters. The data provided can also be useful for the comparison with other groups of trained recreational cyclists. A limitation of the present study is a lack of control on the diet of the subjects during the study period, which could have affected some hematological parameters. Further studies on recreational cyclists, should clarify the effective exercise protocols able to improve the lipid profile.

\section{References}

1. Sarzynski MA, Burton J, Rankinen T, Blair SN, Church TS, Després JP, et. al. The effects of exercise on the lipoprotein subclass profile: A meta-analysis of 10 interventions. Atherosclerosis. 2015; 243(2), 364-372.

2. Foran SE, Lewandrowski KB, Kratz A. Effects Of Exercise On Laboratory Test Results. Laboratory medicine, 2003; 10(34), 736-742.

3. Søndergaard E, Poulsen MK, Jensen MD, Nielsen S. Acute changes in lipoprotein subclasses during exercise. Metabolism. 2014; 63(1), 61-68.

4. Paoli A, Pacelli QF, Moro T, Marcolin G, Neri M, Battaglia G, et. al. Effects of highintensity circuit training, low-intensity circuit training and endurance training on blood pressure and lipoproteins in middle-aged overweight men. Lipids Health Disease. 2013; $12: 131$.

5. Halbert JA, Silagy CA, Finucane P, Withers RT, Hamdorf PA, Andrews GR. The effectiveness of exercise training in lowering blood pressure: a meta-analysis of randomized controlled trials of 4 weeks or longer. Journal of Human Hypertension. 1997; 11:641-649.

6. Halbert JA, Silagy CA, Finucane P, Withers RT, Hamdorf PA. Exercise training and blood lipids in hyperlipidemic and normolipidemic adults: a meta-analysis of randomized, controlled trials. European Journal of Clinical Nutrition. 1999; 53:514-522.

7. Jastrzebska M, Kaczmarczyk M, Suárez AD, Sánchez GFL, Jastrzebska J, Radziminski L, et al. Hematological Parameters and Blood Plasma Lipid Profile in Vitamin D Supplemented and Non-Supplemented Young Soccer Players Subjected to High-Intensity Interval Training. The Journal of Nutritional Science and Vitaminology. 2017; 63(6), 357-364.

8. Tambalis K, Panagiotakos DB, Kavouras SA, Sidossis LS. Responses of blood lipids to aerobic, resistance, and combined aerobic with resistance exercise training: a systematic review of current evidence. Angiology, 2009; 60(5), 614-632. 
9. Siegel AJ, Stec JJ, Lipinska I, Van Cott EM, Lewandrowski KB, Ridker PM et. al. Effect of marathon running on inflammatory and hemostatic markers. American Journal of Cardiology. 2001; 88(8), 918-920, A9.

10. Adner MM, Gembarowicz R, Casey J, Kelley R, Fortin R, Calflin K, et. al. Point-ofCare Biochemical Monitoring of Boston Marathon Runners: A Comparison of Prerace and Postrace Controls to Runners Requiring On-site Medical Attention. Point of Care: The Journal of Near-Patient Testing \& Technology, 2002; 1(4)237-240.

11. Gilli P, De Paoli Vitali E, Tataranni G, Farinelli A. Exercise-induced urinary abnormalities in long distance runners. International Journal of Sports Medicine, 1984; 05(5), 237-240.

12. Banfi G, Colombini A, Lombardi G, Lubkowska A. Metabolic markers in sports medicine. Advances In Clinical Chemistry, 2012; 56:1-54.

13. Priego QJI, Kerr ZY, Bertucci WM, Carpes FP. The categorization of amateur cyclists as research participants: findings from an observational study. Journal of Sports Sciences. $2018 ; 36: 2018-2024$.

14. Borg G. Physical training, perceived exertion in physical work. Lakartidningen, 1970; 67(40), 4548-4557.

15. Böning D, Tibes U, Schweigart U. Red cell hemoglobin, hydrogen ion and electrolyte concentrations during exercise in trained and untrained subjects. European Journal of Applied Physiology, 1976; 35(4), 243-249.

16. Ketelhut RG, Ketelhut K, Messerli FH, Badtke G. Fitness in the fit: Does physical conditioning affect cardiovascular risk factors in middle-aged marathon runners? European Heart Journal, 1996; 17(2), 199-203.

17. Cullinane E, Siconolfi S, Saritelli A, Thompson PD. Acute decrease in serum triglycerides with exercise: is there a threshold for an exercise effect? Metabolism. 1982; 31(8), 844-847.

18. Kratz A, Lewandrowski KB, Siegel AJ, Chun KY, Flood JG, Van Cott EM, et. al. Effect of marathon running on hematologic and biochemical laboratory parameters, including cardiac markers. American Journal Of Clinical Pathology. 2002; 118(6), 856-863.

19. Casoni I, Borsetto C, Cavicchi A, Martinelli S, Conconi F. Reduced hemoglobin concentration and red cell hemoglobinization in Italian marathon and ultramarathon runners. International Journal of Sports Medicine, 1985; 6(03), 176-179. 
20. Wells CL, Stern JR, Hecht LH. Hematological changes following a marathon race in male and female runners. European Journal of Applied Physiology and Occupational Physiology. 1982; 48(1), 41-49.

22. Mairbäurl H. Red blood cells in sports: effects of exercise and training on oxygen supply by

\section{Notes}

The authors have no conflicts of interest.

\section{Acknowledgements}

We want to express our most sincere sympathies to our colleague Andrea Sapone who passed away, and his family. Professor Sapone A., who has collaborated with us during this study, was a highly valuable and respected member of our team

MAX G-0 G-28

Total group $\quad 14,63 \pm 3 \quad 9,65 \pm 2,74 \quad 7,35 \pm 3^{*}$

Tab. 1. Max Blood lactate (mM/L) measured during the ramp test, after exhaustion test at baseline (G-0) , (G-1) and after 28 days (G-28) of training, in all subjects * $\mathrm{p}=0,14$;

$\begin{array}{lrrr}\text { Index } & \text { PRE } & \text { POST } & \text { p value } \\ \text { GLI } & 80,95 \pm 15,59 & 98,30 \pm 14,79 & 0,001^{*} \\ \text { YGT } & 19,79 \pm 7,33 & 21,21 \pm 6,77 & 0,000^{*} \\ \text { GOT } & 26,20 \pm 8,10 & 34,45 \pm 8,95 & 0,000^{*} \\ \text { GPT } & 23,40 \pm 11,13 & 40,80 \pm 15,16 & 0,000^{*} \\ \text { HDL } & 62,14 \pm 15,45 & 67,84 \pm 18,45 & 0,000^{*}\end{array}$




$\begin{array}{lccc}\text { CHOL } & 182,55 \pm 22,47 & 190,70 \pm 33,55 & 0,093 \\ \text { TG } & 81,20 \pm 49,67 & 84,70 \pm 32,95 & 0,269 \\ \text { LDL } & 88,64 \pm 16,89 & 94,86 \pm 21,23 & 0,010^{*} \\ \text { RBC } & 4,85 \pm 0,39 & 5,11 \pm 0,35 & 0,000^{*} \\ \text { HGB } & 14,77 \pm 0,92 & 15,36 \pm 0,85 & 0,000^{*} \\ \text { HCT } & 45,11 \pm 3,08 & 47,31 \pm 2,61 & 0,000^{*} \\ \text { MCV } & 92,71 \pm 4,34 & 92,79 \pm 4,35 & 0,356 \\ \text { MCH } & 30,39 \pm 1,53 & 30,13 \pm 1,34 & 0,016^{*} \\ \text { RDW } & 13,26 \pm 0,80 & 13,3 \pm 30,82 & 0,104 \\ \text { WBC } & 6,27 \pm 2,34 & 9,01 \pm 3,63 & 0,000^{*}\end{array}$

274 Tab 2. Ematochemistry PRE-POST 1 hour intensive cycling on the cycloergometer in the total sample.(G 0).

275 GLI: Glicemia, mg/dl; YGT, GOT and GPT: Transamynases, U/L; HDL: HDL-Cholesterol, mg/dl; CHOL: 276 total Cholesterol, mg/dl; TRI: Tryglicerides mg/dl; LDL: LDL-Cholesterol, mg/dl.RBC : Red Blood Cell , 277 10^6/ul ; HGB: Hemoglobin, g/dl; HCT: Hematocrit, \%; MCV Mean Corpuscular Volume, fl; MCH: Mean 278 Content of Hemoglobin, pg; MCHC: Mean Concentration of Hemoglobin Content, g/dl; RDW: Red Blood 279 Cell Distribution Wide, \%; WBC : White blood cell, 10^3/ul. * significantly different 
LDL $\quad 88,64 \pm 16,89 \quad 83,32 \pm 17,33 \quad 0,083 *$

298

$299 \mathrm{RBC} \quad 4,84 \pm 0,37 \quad 4,86 \pm 0,42 \quad 0,827$

300

301

HGB $\quad 14,71 \pm 0,91$

$14,79 \pm 1,15 \quad 0,888$

302

303

HCT $\quad 44,96 \pm 3,06 \quad 45,13 \pm 3,56 \quad 0,952$

304

305

$\mathrm{MCV} \quad 92,52 \pm 4,30$

$93,04 \pm 4,44$

$0,09 *$

306

307

MHC $\quad 30,3 \pm 1,51$

$30,49 \pm 1,51$

0,462

308

309

MCHC $32,75 \pm 0,57$

$32,77 \pm 0,64$

0,87

310

311

RDW $13,29 \pm 0,79$

$13,26 \pm 0,86$

0,969

312

313

WBC $\quad 6,27 \pm 2,34 \quad 6,01 \pm 1,46 \quad 0,604$

314

315

316

317

318

319

320

Tab. 3. Ematochemical parameters at G0 and G28, in the pre-TGal condition. * significantly different

GLI: Glicemia, mg/dl; YGT, GOT and GPT: Transamynases, U/L; HDL: HDL-Cholesterol, mg/dl; CHOL: total Cholesterol, mg/dl; TRI: Tryglicerides mg/dl; LDL: LDL-Cholesterol, mg/dl.RBC : Red Blood Cell , 10^6/ul ; HGB: Hemoglobin, g/dl; HCT:

Hematocrite, \%; MCV Mean Corpuscular Volume, fl; MCH: Mean Content of Hemoglobin, pg; MCHC: Mean Concentration of Hemoglobin Content, g/dl; RDW: Red Blood Cell Distribution Wide, \%; WBC : White blood cell, 10^3/ul. * significantly different 